\title{
The transcription factor Zic4 acts as a transdifferentiation switch
}

Matthias Christian Vogg ${ }^{1}$, Jaroslav Ferenc ${ }^{2,3}$, Wanda Christa Buzgariu ${ }^{1}$, Chrystelle Perruchoud $^{1}$, Panagiotis Papasaikas ${ }^{2,4}$, Paul Gerald Layague Sanchez ${ }^{1}$, Clara Nuninger ${ }^{2,3}$, Céline Delucinge-Vivier ${ }^{5}$, Christine Rampon ${ }^{6}$, Leonardo Beccari ${ }^{7}$, Sophie Vriz ${ }^{6}$, Stéphane Vincent $^{8}$, Brigitte Galliot ${ }^{1, *}$, Charisios D. Tsiairis ${ }^{2, *}$

\section{Affiliations:}

${ }^{1}$ Department of Genetics and Evolution, Institute of Genetics and Genomics (iGE3), Faculty of Sciences, University of Geneva; 30 Quai Ernest Ansermet, 1211 Geneva 4, Switzerland.

${ }^{2}$ Friedrich Miescher Institute for Biomedical Research; Maulbeerstrasse 66, 4058 Basel, Switzerland.

${ }^{3}$ University of Basel; Petersplatz 1, 4001 Basel, Switzerland

${ }^{4}$ SIB Swiss Institute of Bioinformatics; 4058 Basel, Switzerland

${ }_{5}^{5}$ iGE3 Genomics Platform, University of Geneva; 1 Rue Michel-Servet, 1211 Geneva 4, Switzerland.

${ }^{6}$ Centre Interdisciplinaire de Recherche en Biologie (CIRB), CNRS UMR 7241/INSERM U1050/Collège de France; 11 Place Marcelin Berthelot, 75231 Paris Cedex 05, France.

${ }^{7}$ Institut NeuroMyoGène, CNRS UMR 5310, INSERM U1217, University Claude Bernard Lyon1; Lyon, France.

${ }^{8}$ LBMC, UMR 5239 CNRS/Ecole Normale Supérieure de Lyon, SFR 128 Biosciences Lyon Gerland, Université de Lyon ; 69007 Lyon, France.

*Corresponding author. Email: charisios.tsiairis@,fmi.ch; brigitte.galliot@unige.ch 


\begin{abstract}
:
The molecular mechanisms that maintain cell identities and prevent transdifferentiation remain mysterious. Interestingly, both dedifferentiation and transdifferentiation are transiently reshuffled during regeneration. Therefore, organisms that regenerate readily offer a fruitful paradigm to investigate the regulation of cell fate stability. Here, we used Hydra as a model system and show that Zic4 silencing is sufficient to induce transdifferentiation of tentacle into foot cells. We identified a Wnt-controlled Gene Regulatory Network that controls a transcriptional switch of cell identity. Furthermore, we show that this switch also controls the re-entry into the cell cycle. Our data indicate that maintenance of cell fate by a Wnt-controlled GRN is a key mechanism during both homeostasis and regeneration.
\end{abstract}

\title{
One-Sentence Summary:
}

A Wnt-controlled GRN controls fate maintenance in Hydra.

\section{Main Text:}

When cells differentiate during embryonic development or adult regeneration, they acquire specific characteristics, a specialization compensated by a loss of plasticity (Lambert et al. 2021). The protection of the identity of terminally differentiated cells relies on mechanisms that are poorly understood. Therefore, model systems that readily reactivate developmental processes such as the freshwater polyp Hydra, help decipher the mechanisms underlying the balance between plastic cell fates and stable cell identities. The animals, which can regenerate any missing body part after amputation, are composed of two epithelial cell layers, the epidermis and the gastrodermis, which in the body column are populated with three distinct stem cell populations, the epidermal and gastrodermal epithelial stem cells (ESCs), and the multipotent interstitial stem cells (ISCs) (Vogg et al. 2021). The ESC populations proliferate continuously in the body column, and terminally differentiate when they reach the apical (head) or basal (foot) regions. The tip of the head, named hypostome, harbors a head organizer required to maintain apical patterning in intact animals, an activity positively regulated by $\mathrm{Wnt} 3 / \beta$-catenin signaling and restricted by the transcription factor Sp5 (Nakamura et al. 2011)(Vogg et al. 2019) (Figure 1A). When Sp5 expression is knockeddown, Wnt3 is de-repressed along the body column and ectopic head formation occurs. The base of the head is surrounded by tentacles where the epidermal layer is made of large epithelial cells arrested in G2 and terminally differentiated as tentacle battery cells (TBC) (Figure Supplement 
1). Each TBC contains approximately twenty mechano-sensory cells named nematocytes (or stinging cells). These cells discharge a venom capsule named nematocyst when a specialized cilium, the cnidocil, is stimulated by prey (Holstein 2012). At the other extremity of the animal, the epidermal epithelial cells of the basal disc (foot) named basal disc cells (BDC) are terminally differentiated and produce acid mucopolysaccharide (MPS) droplets that facilitate the attachment to substrates (Rodrigues et al. 2016). Thus, both the tentacles and the foot display highly differentiated cells. Whether terminally differentiated epithelial cells can acquire an alternative identity in Hydra is unknown. Here, we show that the silencing of the transcription factor Zic4 is sufficient to induce transdifferentiation of tentacle battery cells into basal disc cells.

Among 83 putative Sp5 target genes previously identified in HEK293T cells (Vogg et al. 2019), we focused in the zinc-finger transcription factor Zic4 that displays an apical-to-basal graded expression along the Hydra body axis. Interestingly, Zic4 is predominantly expressed in the tentacle zone of intact animals and re-expressed within 16 hours after amputation in apicalregenerating tissues (Figure 1A, Figure Supplement 2-3, Dataset Supplement 1). Amongst the four Hydra Zic-related genes, Zic4 is the only one to show these features (Figure Supplement 45). Zic4 appears positively regulated by $S p 5$ and $\mathrm{Wnt} / \beta$-catenin signaling as its expression is notably reduced in the tentacle zone after Sp5 or $\beta$-catenin silencing (Figure 1B-1D, Figure Supplement 6). When Wnt/ $\beta$-catenin signaling is ubiquitously activated by Alsterpaullone (ALP) treatment, a dual regulation is observed for Zic4, a down-regulation in the apical region together with a global up-regulation in the body-column after two days. This turns into an "Octopus" phenotype after four days, i.e. multiple Zic4-expressing rings, with each ring corresponding to the base of an ectopic tentacle (Figure 1E, Figure Supplement 7). This regulation is likely direct as the 3'505 bp Zic4 upstream sequence contains four consensus TCF/LEF binding sites (Figure Supplement 8). When expressed in HEK293T cells, the Zic4-3505:luciferase construct is upregulated when human $\beta$-catenin, Wnt3 or LRP6 are overexpressed (Figure 1F). In zebrafish embryos the overexpression of HyZic4 induces Wnt-like phenotypes, namely eye and axial anomalies, which reflect inappropriate Wnt/ $\beta$-catenin signaling (Figure Supplement 9). To assess Zic4 regulation in vivo, we produced a Zic4-3505:GFP transgenic line, and recorded an increase in GFP expression following ALP treatment (Figure 1G). These data demonstrate a positive regulation of $\mathrm{Wnt} / \beta$-catenin signaling and $\mathrm{Sp} 5$ on Zic4 expression in the tentacle zone in homeostatic conditions, and along the body column when $\mathrm{Wnt} / \beta$-catenin signaling is up-regulated. 
To explore Zic4 function, we knocked-down Zic4 by RNA interference (RNAi). Intact Zic4(RNAi) animals exhibit tentacles with half the length of those in control animals. The overall tentacle number is not affected $(100 \% ; n=100)$ (Figure 2A, Figure Supplement 10A). In contrast, apicalregenerating Zic4(RNAi) animals, regenerate shorter but also $25 \%$ fewer tentacles $(100 \% ; n=99)$ (Figure 2B, Figure Supplement 10B). We also knocked-down Zic4 in ALP-treated Hydra and found the development of ectopic tentacles along the body column strongly impaired (Figure 2C, Figure Supplement 10C). Consistent with a role in tentacle formation, we also observed ectopic Zic4 expression in the second tentacle ring induced by DAC-2-25 treatment (Glauber et al. 2013) (Figure Supplement 11). We also noticed that the expression of two components of the head organizer, Wnt 3 and Bral, is not affected in intact or regenerating Zic4(RNAi) animals, suggesting that Zic4 is required neither for the maintenance nor for the formation of a functional head organizer (Figure 2D, Figure Supplement 12). Indeed, when we transplanted apical tissue from control and Zic4(RNAi) animals into actin:GFP transgenic animals, we observed a similar induction of a secondary body axis, a hallmark of a functional head organizer (Figure Supplement 13). We concluded that Zic4 is required for tentacle maintenance and tentacle formation.

Next, we searched for changes underlying deficient tentacle maintenance and tentacle formation in Zic4(RNAi) animals. To monitor the cellular composition of tentacles, we used transgenic FUCCI-Hydra that constitutively express GFP in epidermal epithelial cells and noted a two-fold decrease in the TBC number after Zic4(RNAi) (Figure 2E, Figure Supplement 14-15). Nevertheless, we found the levels of epithelial proliferation and epithelial apoptosis unchanged after Zic4 or Zic4/Sp5 knock-down (Figure Supplement 16). Similarly, we did not record any change in the migration/displacement behavior of cells towards the head region (Figure Supplement 17). The RNA-seq analysis performed on scrambled and Zic4(RNAi) animals (Figure 2F, Dataset Supplement 2) reveals a striking decrease in the transcript level of three tentacle markers ksl, nematocilin-A, nematocilin-B (Hwang et al. 2008)(Hwang et al. 2007), consistent with the Zic4(RNAi)-induced loss of TBCs. Furthermore, we noted an up-regulation of genes normally expressed in the basal disc, including the peroxidases PPOD1, PPOD2, PPOD2l, PPOD11, the homeogenes Dlx 1 and NK2, the transmembrane BMP regulator Crim-1 and the BMP antagonist NBL1 (Wenger et al. 2019) (Figure 2F, Table Supplement 2). These findings suggest that Zic4 controls tentacle identity by repressing basal gene expression. 
In Zic4(RNAi), Sp5(RNAi) and Zic4/Sp5(RNAi) animals, we found tentacle regions that express the basal marker Crim-1 but no longer Nematocilin-A, as well as ectopic structures along the body column that do not express Wnt3 but express Crim-1 three days post-EP3 (Figure 3A, Figure Supplement 18-21). In the same conditions, we observed MPS droplets typical of basal disc cells (BDC) in tentacle cells as well as in the structures ectopically formed along the body column when Zic4 and/or Sp5 are knocked-down (Figure 3B, Figure Supplement 20-21). This phenotype is enhanced in Zic4/Sp5(RNAi) animals that develop complete ectopic basal discs, which are morphologically indistinguishable from those of control animals $(100 \%, n=70)$. We observed a similar tentacle to basal disc transformation upon $\beta$-catenin silencing (Figure Supplement 22), likely as a result of Zic4 down-regulation. This tissue transformation exhibits a stronger penetrance in $H v_{-}$AEP than in $H v_{-}$Basel animals (Figure Supplement 18-23).

We hypothesized that the formation of ectopic basal discs relies on the transdifferentiation of TBCs. As supporting evidence, we found TBCs, which exhibit a mixed identity, half tentacle, half basal disc three days post-EP3 with the typical basal mucus aspect in the close vicinity of degenerating nematocytes (Figure 3C, Supplement Movie 1-3). These TBCs actually contain lipid droplets with peroxidase activity, never observed in apical regions of scramble(RNAi) animals (Figure 3D). Four days later, Zic4/Sp5(RNAi) animals harbor tentacles fully transformed into basal discs characterized by an intense peroxidase activity (Figure Supplement 24). This transformation occurs already after two exposures to Zic4/Sp5 siRNAs, as evidenced by tentacles containing two days post-EP2 TBCs with degenerated nematocytes, a typical granular cytoplasm (Figure 3E, Figure Supplement 25, Supplement Movie 4-5) and peroxidase+ droplets (Figure Supplement 26). We confirmed the early start of this transdifferentiation event by identifying in apical tissues macerated two days post-EP2 isolated TBCs that contain nematocytes expressing nematocilin- $A$ together with the BDC marker Crim-1 (Figure 3F, Figure Supplement 27), or isolated TBCs that contain droplets with peroxidase activity (Figure 3G, Figure Supplement 28). These results confirm the transdifferentiation process, i.e. TBCs that transiently co-express tentacle and basal disc markers.

To further investigate gene-expression changes that occur in tentacles of animals knocked-down for Zic4 and/or Sp5, we used proximal and basal tentacle tissue for RNA-seq analysis. When projected into a PCA space, generated from the positional sequencing of different body parts, the overall identity of the RNAi tentacles samples seems to shift only marginally from the location of 
the intact tentacles (Figure 4A, Figure Supplement 29, Dataset Supplement 3-4). However, when the identity map is constructed with epithelia-specific genes, we noted a clear shift from tentacle towards basal identity in all RNAi samples. All investigated conditions show an increase in the expression of basal markers, combined with a decrease of tentacle marker genes (Figure 4B, Dataset Supplement 4), with the strongest modulations in the tentacles of Zic4/Sp5(RNAi) animals but also in ectopic structures of $S p 5$ (RNAi) animals. These results, which confirm the basal transformation of TBCs, suggest a key role for Zic4 as we recorded the highest level of Zic4 silencing after Zic4/Sp5(RNAi) when compared to Sp5(RNAi) or Zic4(RNAi) (Figure Supplement 30).

Next, we investigated whether cells that show signs of transdifferentiation also re-enter the cell cycle. First, we analyzed tentacles of FUCCI-eGFP Hydra knocked-down twice for Zic4/Sp5 and we noted in cells that contain peroxidase+ droplets, a change from red/pink to yellow nuclear fluorescence, suggesting a progression through S/G2 phase (Figure 4C, Figure Supplement 31). To confirm cell cycle re-entry, we performed a 5-hour BrdU labeling of FUCCI-eGFP Hydra knocked-down for Zic4/Sp5. Two days post-EP2, we identified in tentacles BrdU+ nuclei among TBCs (Figure 4D, Figure Supplement 32), peroxidase+ droplets in BrdU+ TBCs (Figure 4E, Figure Supplement 33B), Crim-1 expression in BrdU+ TBCs (Figure 4F, Figure Supplement 33A) and finally an increase in the number of BrdU+ apical epithelial cells quantified on macerated tissues or on whole-mounts (Figure 4G, Figure Supplement 34) but no increase in the number of dividing cells (Figure Supplement 35). We found that after four days of Zic4/Sp5 silencing, the typical organization of TBCs becomes disrupted in the proximal part of tentacles of several animals, with a sharp boundary with the distal part that remain well organized (Figure 4E, Figure Supplement 34).

To collect evidence on the molecular mechanism that possibly drives cell-cycle re-entry, we analyzed the GO term of the genes consistently upregulated when Zic4 and/or Sp5 are knockeddown and we identified biological functions linked to cell cycle, i.e. G1/S transition, positive regulation of the cell cycle, chromosome segregation (Figure 4H, Dataset Supplement 5), confirming that epithelial cell cycling is enhanced upon Zic4 and/or Sp5 silencing. To test whether cell cycling is necessary for tentacle transdifferentiation, we inhibited DNA synthesis with two pulses of Hydroxyurea (HU). We observed that ectopic Crim-1 spots no longer form in tentacles of Zic4/Sp5(RNAi) animals, while apical Crim-1 expression is transiently abrogated, in agreement 
with the transient HU-induced blockade of cell cycle progression (Buzgariu et al. 2018) (Figure 4I, Figure Supplement 36). Taken together, these data indicate that upon Zic4/Sp5 silencing, TBCs do not maintain their typical tentacle character, re-enter the cell cycle but do not divide, and fully transform within less than seven days into basal disc cells.

The results obtained in intact and regenerating animals indicate that distinct levels of Wnt/ $\beta$ catenin signaling, Sp5 and Zic4 activity define three regions in the apical region and trigger the formation of two distinct structures, the hypostome and the tentacle zone. In the hypostome Wnt/ $\beta$ catenin signaling is high, while Zic4 and Sp5 are low. The tentacle zone is characterized by low Wnt/ $\beta$-catenin signaling and high Sp5, inducing Zic4 expression and thus TBC differentiation and tentacle formation. The enhanced transdifferentiation phenotype obtained in animals submitted to the double Zic4/Sp5 knockdown is coherent with an epistatic relationship between Sp5 and Zic4. We conclude that Zic4 acts as a master regulator to control the choice between two epidermal cell fates, maintenance or differentiation of battery cells when Zic4 is high, differentiation of basal mucous cells when Zic4 is low. Therefore, we suspect Zic4 to repress the basal disc cell status, which appears as a default state of epithelial epidermal differentiation, possibly reflecting an ancestral differentiation fate of pre-bilaterian multifunctional epithelial stem cells. In the sea anemone Nematostella, several Zic genes are expressed during tentacle formation in distinct tentacle cell types (Layden et al. 2010), suggesting a function in tentacle formation possibly shared among cnidarians.

We also present evidence for epithelial transdifferentiation in Hydra, with a model where low levels of expression of the Zic4 and Sp5 transcription factors lead to cell fate change. In such context, clustered tentacle battery cells first dedifferentiate, namely lose $k s 1$ expression, no longer maintain embedded nematocytes as evidenced by the loss of NemA expression, while the typical tentacle architecture gets disorganized as the large extra-cellular space between TBCs disappears. In parallel, these TBCs re-enter the cell cycle without dividing and re-differentiate into basal disc cells that express Crim-1, differentiate droplets with peroxidase activity at the apical pole first and rapidly form a compact mass (Figure 4J, Figure Supplement 37). As TBCs that express both tentacle and basal markers could be identified, we concluded that this process fulfills the criteria of transdifferentiation (Lambert et al. 2021). In addition, cell division was not observed among tentacle BrdU-positive cells, and mitotic activity not detected in tentacles, therefore, we conclude that cells that re-enter S-phase undergo endoreplication, a process observed in developmental as 
well as injury and stress contexts, associated with regeneration (Lee et al. 2009)(Nandakumar et al. 2021).

Zinc finger transcription factors (ZF-TF) play a crucial role in cell fate stability, the ectopic expression of a single ZF-TF (Zfp521) being able to promote the conversion of human fibroblasts to neural stem cells ex vivo (Shahbazi et al. 2016). Among them, the Zic family members act as versatile multifunctional proteins, classical DNA-binding proteins that regulate the expression of the ascidian Brachyury or the mammalian Oct4 and Nanog via their promoter sequences. Zic genes also act as transcriptional co-activators via their protein-protein interaction with a variety of transcription factors such as Gli, TCF, Smad, Pax, Cdx, SRF as well as chromatin remodeling factors contributing to enhancer functions (Hatayama and Aruga 2018). Of interest, in vertebrates, Zic proteins inhibit Wnt/ $\beta$-catenin signaling (Fujimi et al. 2012), compete with Sp transcription factors on promoter sequences (Yang et al. 2000), or associate with Geminin in cell cycle regulation (Sankar et al. 2016). The current identification of a Zic4/Wnt-dependent GRN that acts as a switch regulating the fate of epithelial cells, opens the way for deeper investigation of this GRN in evolutionary-distant eumetazoan phyla.

\section{References:}

Buzgariu W, Wenger Y, Tcaciuc N, Catunda-Lemos A-P, Galliot B. 2018. Impact of cycling cells and cell cycle regulation on Hydra regeneration. Dev Biol 433:240-253.

Fujimi TJ, Hatayama M, Aruga J. 2012. Xenopus Zic3 controls notochord and organizer development through suppression of the Wnt/ $\beta$-catenin signaling pathway. Dev Biol 361:220-231.

Glauber KM, Dana CE, Park SS, Colby DA, Noro Y, Fujisawa T, Chamberlin AR, Steele RE. 2013. A small molecule screen identifies a novel compound that induces a homeotic transformation in Hydra. Development 140:4788-4796.

Hatayama M, Aruga J. 2018. Role of Zic Family Proteins in Transcriptional Regulation and Chromatin Remodeling. Adv Exp Med Biol 1046:353-380.

Holstein TW. 2012. A view to kill. BMC Biol 10:18.

Hwang JS, Ohyanagi H, Hayakawa S, Osato N, Nishimiya-Fujisawa C, Ikeo K, David CN, Fujisawa T, Gojobori T. 2007. The evolutionary emergence of cell type-specific genes inferred from the gene expression analysis of Hydra. Proc Natl Acad Sci US A 104:14735-14740. 
Hwang JS, Takaku Y, Chapman J, Ikeo K, David CN, Gojobori T. 2008. Cilium evolution: identification of a novel protein, nematocilin, in the mechanosensory cilium of Hydra nematocytes. Mol Biol Evol 25:2009-2017.

Lambert J, Lloret-Fernández C, Laplane L, Poole RJ, Jarriault S. 2021. On the origins and conceptual frameworks of natural plasticity-Lessons from single-cell models in C. elegans. Curr Top Dev Biol 144:111-159.

Layden MJ, Meyer NP, Pang K, Seaver EC, Martindale MQ. 2010. Expression and phylogenetic analysis of the zic gene family in the evolution and development of metazoans. Evodevo $1: 12$.

Lee HO, Davidson JM, Duronio RJ. 2009. Endoreplication: polyploidy with purpose. Genes Dev 23:2461-2477.

Nakamura Y, Tsiairis CD, Özbek S, Holstein TW. 2011. Autoregulatory and repressive inputs localize Hydra Wnt3 to the head organizer. Proc Natl Acad Sci U S A 108:9137-9142.

Nandakumar S, Rozich E, Buttitta L. 2021. Cell Cycle Re-entry in the Nervous System: From Polyploidy to Neurodegeneration. Front Cell Dev Biol 9:698661.

Rodrigues M, Leclère P, Flammang P, Hess MW, Salvenmoser W, Hobmayer B, Ladurner P. 2016. The cellular basis of bioadhesion of the freshwater polyp Hydra. BMC Zool 1:3.

Sankar S, Yellajoshyula D, Zhang B, Teets B, Rockweiler N, Kroll KL. 2016. Gene regulatory networks in neural cell fate acquisition from genome-wide chromatin association of Geminin and Zic1. Sci Rep 6:37412.

Shahbazi E, Moradi S, Nemati S, Satarian L, Basiri M, Gourabi H, Zare Mehrjardi N, Günther P, Lampert A, Händler K, et al. 2016. Conversion of Human Fibroblasts to Stably SelfRenewing Neural Stem Cells with a Single Zinc-Finger Transcription Factor. Stem Cell Reports 6:539-551.

Vogg MC, Beccari L, Iglesias Ollé L, Rampon C, Vriz S, Perruchoud C, Wenger Y, Galliot B. 2019. An evolutionarily-conserved Wnt3/ $\beta$-catenin/Sp5 feedback loop restricts head organizer activity in Hydra. Nat Commun 10:312.

Vogg MC, Buzgariu W, Suknovic NS, Galliot B. 2021. Cellular, Metabolic, and Developmental Dimensions of Whole-Body Regeneration in Hydra. Cold Spring Harb Perspect Biol 13:a040725.

Wenger Y, Buzgariu W, Perruchoud C, Loichot G, Galliot B. 2019. Generic and contextdependent gene modulations during Hydra whole body regeneration. Developmental Biology Available from: http://biorxiv.org/lookup/doi/10.1101/587147

Yang Y, Hwang CK, Junn E, Lee G, Mouradian MM. 2000. ZIC2 and Sp3 repress Sp1-induced activation of the human D1A dopamine receptor gene. J Biol Chem 275:38863-38869. 
Acknowledgments: We thank all members of the Tsiairis and Galliot lab for discussions, Denis Duboule for comments on the manuscript. The authors thank the iGE3 Genomic Platform for RNA-seq library preparation and sequencing, the FMI Genomics facility and Sebastien Smallwood for RNA sequencing experiments, the FMI Imaging facility for support with microscopy, and Iskra Katic for Hydra electroporations.

\section{Funding:}

Swiss National Foundation grants 31003_169930 and 310030_189122; Swiss Government Excellence Scholarships for Foreign Scholars; Novartis Foundation.

Author contributions: Conceptualization: MCV, JF, SVi, BG, CDT; Methodology: MCV, JF, WB, BG, CDT; Investigation: MCV, JF, WB, CP, PP, PGLS, CN, CD, CR, SVr; Visualization: MCV, JF, WB, PGLS, LB, BG, CDT; Funding acquisition: BG, CDT; Supervision: BG, CDT; Writing - original draft: MCV; Writing - review \& editing: BG, MCV, JF, WB, PGLS, SVi, CDT

\section{Competing interests:}

The authors declare no competing interests.

Data availability: The RNA-seq data have been deposited in the GEO database under the accession codes GSE190110 and GSE191177. All other raw data can be found in Dataset Supplement 6. 


\section{Figures \& Legends}

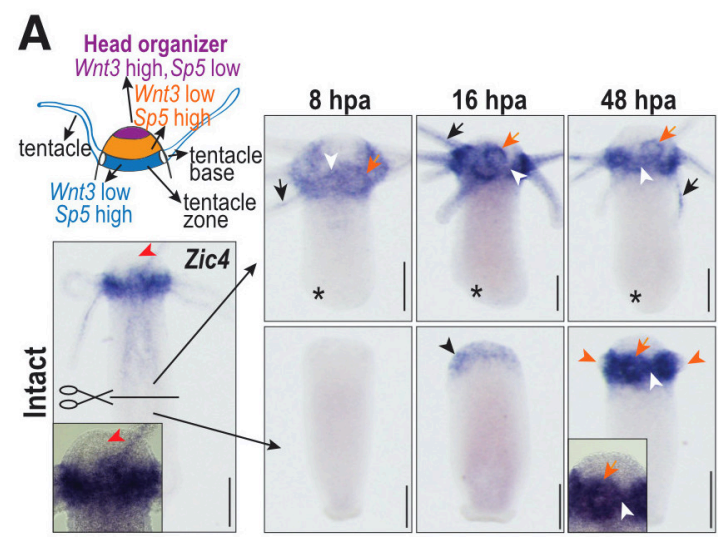

D
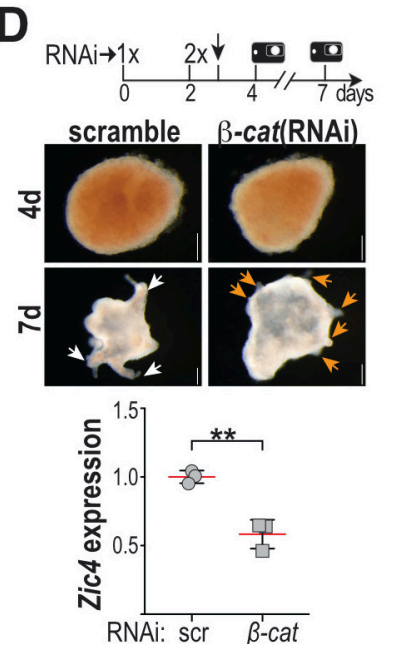

B
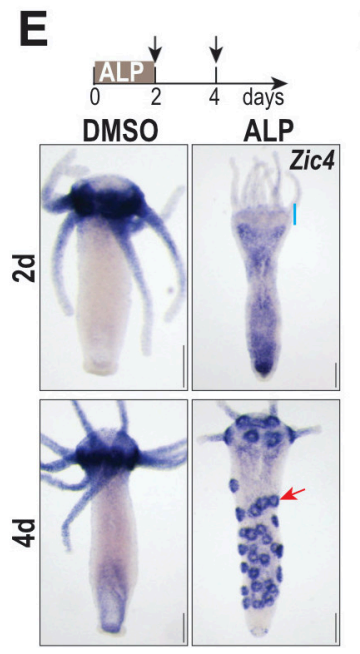
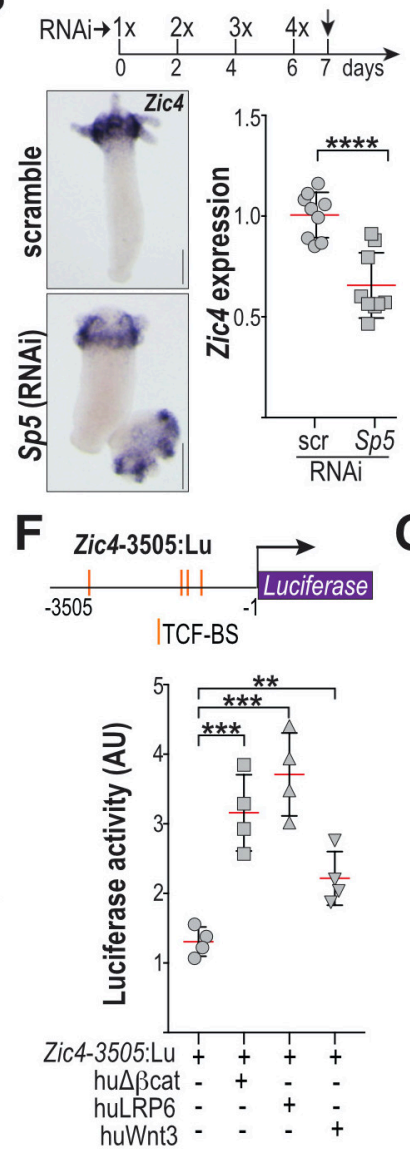

C RNAi $\rightarrow \begin{array}{lllll}1 x & 2 x & 3 x & 4 x \\ 0 & 1 & 1 & 1 & 1 \\ 0 & 2 & 4 & 6 & 7 \text { days }\end{array}$

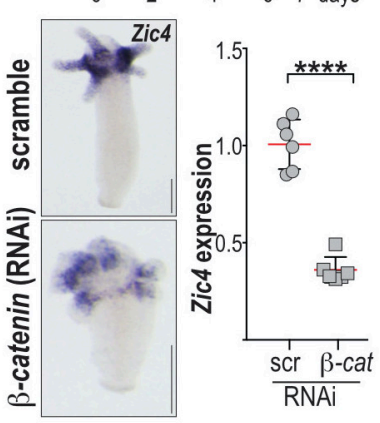

G
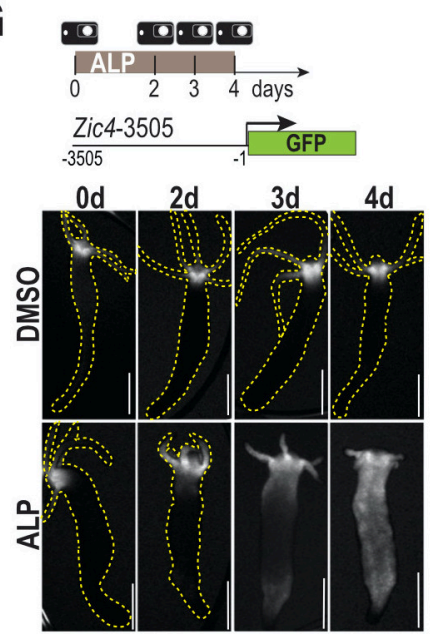

Figure 1. Zic4, a tentacle zone marker, target of Sp5 and Wnt/ $\beta$-catenin signaling.

(A) Scheme depicting Wnt3 and Sp5 apical expression domains. Zic4 expression detected in homeostatic heads: tentacles (black arrows), tentacle base (orange arrows), tentacle zone (white arrowheads), hypostome (red arrowheads), during basal (asterisks) and apical regeneration at 16 hpa (black arrowhead) and 48 hpa (orange arrowheads: tentacle rudiments). (B-C) Zic4 expression detected by ISH and qPCR (d7) after 4 electroporations with scramble (control), Sp 5 or $\beta$-catenin siRNAs. (D) Apical differentiation in reaggregates obtained from animals electroporated twice with scramble or $\beta$-catenin siRNAs before dissociation (black arrow): apical rudiments (orange arrows), fully differentiated heads (white arrows). (Bottom) Zic4 expression measured by qPCR in reaggregates taken at day-7. (E) Ectopic Zic4 expression in ALP-treated animals, reduced at the apex (blue line) at day-2, in tentacle bases (red arrow) at day-4. (F) Luciferase activity driven by the Zic4 promoter in HEK293T cells when $\beta$-catenin signaling is activated. Orange bars: TCF binding-sites (TCF-BS). (G) GFP fluorescence in Zic4-3505:GFP transgenic animals exposed to ALP. In B, C, D and F, each point represents one biological replicate. Statistical p-values: ** $\leq$ $0.01 ; * * * \leq 0.001 ; * * * * \leq 0.0001$. Error bars indicate SD. Scale bars: $200 \mu \mathrm{m}$. 

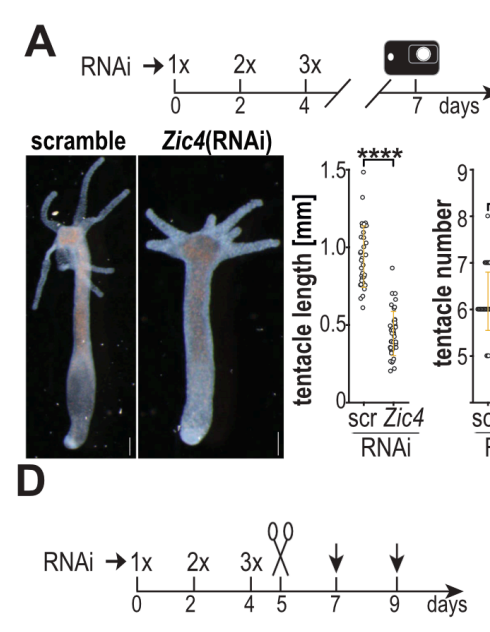

B
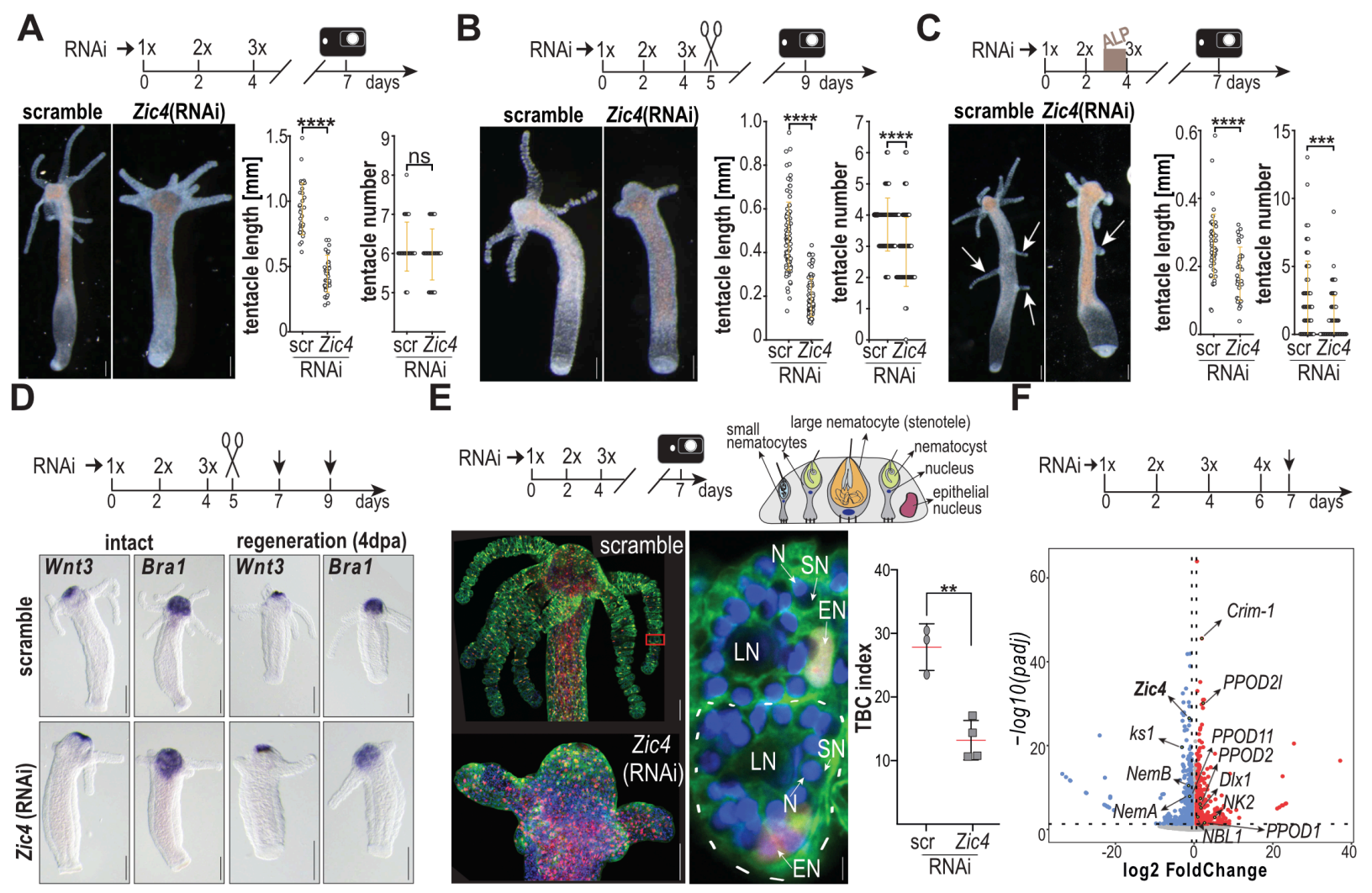

Figure 2. Zic4 is required for Tentacle Battery Cell (TBC) differentiation and tentacle formation.

(A-C) Impact of Zic4 silencing in intact (A), apical-regenerating (B) and ALP-treated (C) Hv_Basel animals. Zic4(RNAi) animals exhibit shorter (A-C) and fewer tentacles (B-C). Each data point represents one animal. White arrows: ectopic tentacles. (D) Wnt3 and Bral expression in intact (left) and head-regenerating (right) Zic4(RNAi) Hv_Basel fixed on d7 (intact) and d9 (apical-regenerating). (E) Schematic view of a tentacle battery cell (TBC) that typically contains one large nematocyte (LN, stenotele) and numerous small nematocytes (SN). Epidermal epithelial cells and TBCs from transgenic FUCCI-eGFP Hydra constitutively express cytoplasmic GFP and nuclear mCherry. Red square: enlarged TBCs, one being outlined with a white dashed line, EN: epithelial nuclei; N: DAPI-stained nuclei. The TBC index corresponds to the number of TBCs per 100 gastrodermal cells counted on macerated apical regions from scramble and Zic4(RNAi) animals. Each point represents one replicate from two independent experiments. (F) Volcano plot representing differentially expressed genes after Zic4(RNAi) as indicated. Statistical p-values: ** $\leq$ $0.01 ; * * * \leq 0.001 ; * * * * \leq 0.0001$. Error bars indicate SDs. Scale bars : $200 \mu \mathrm{m}$ (A-D), $100 \mu \mathrm{m}(\mathrm{E})$, $5 \mu \mathrm{m}$ for enlarged views. 

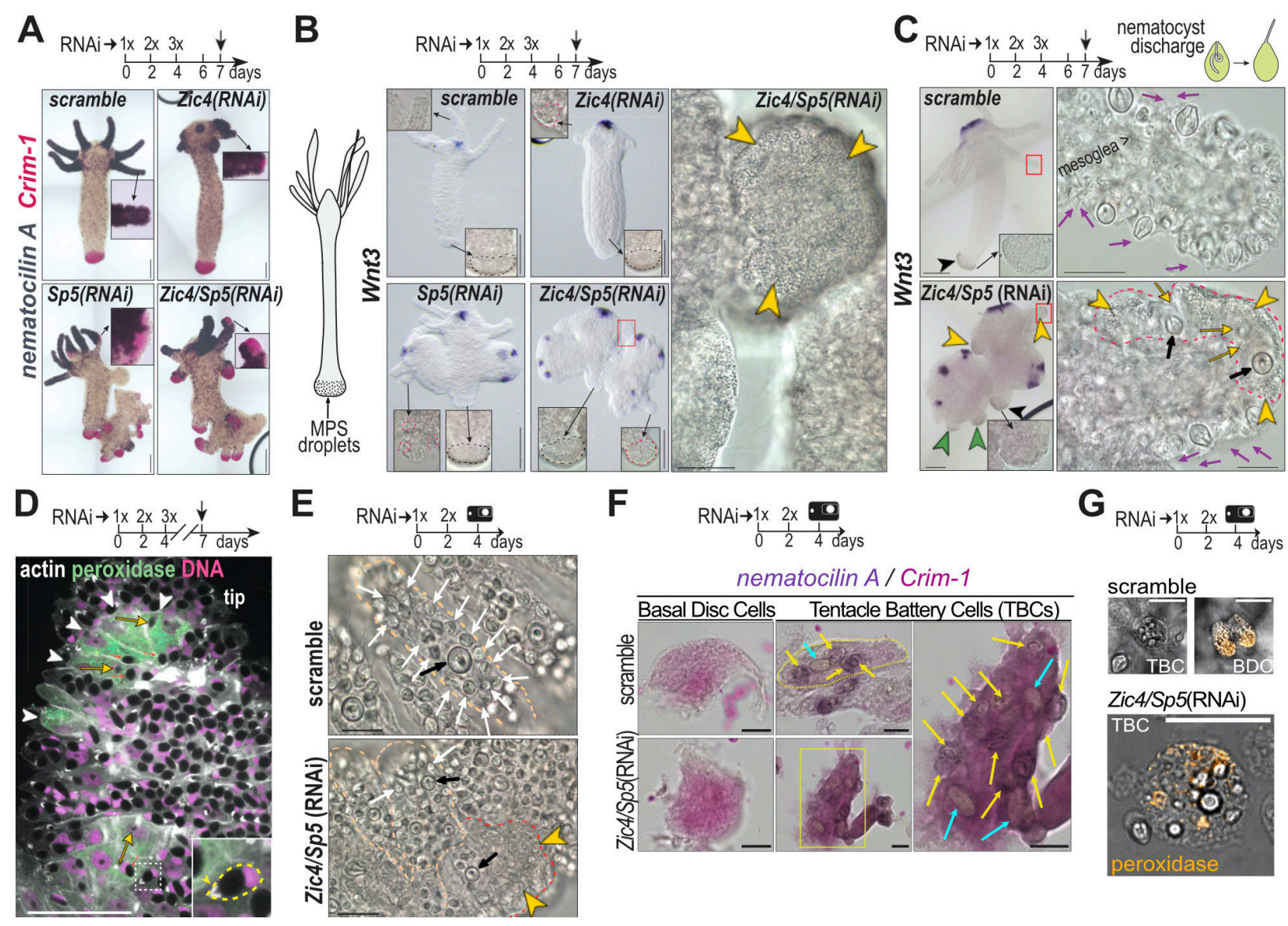

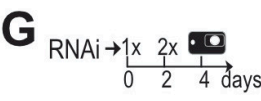

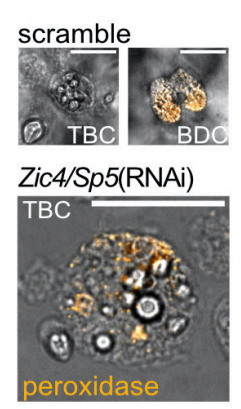

Figure 3. Dedifferentiation and basal differentiation of TBCs in Hydra knocked-down for Zic4 and/or Sp5.

(A) Nematocilin-A (purple) and Crim-1 (pink) expression in $H v_{-} A E P 2$ knocked-down as indicated. (B, C) Wnt 3 expression and ectopic differentiation of basal tissue in $H v \_B a s e l$ knockeddown as indicated. Black outline: original basal discs; red outline: ectopic basal tissue; red squares: enlarged transforming regions; yellow arrowheads: basal-like cells in tentacles characterized by abundant mucopolysaccharide (MPS) droplets; green arrowheads: basal-like cells in ectopic axial structures; purple arrows: discharged nematocysts; yellow arrows: degenerating SNs; black arrows: stenoteles. (D) Zic4/Sp5(RNAi) tentacle containing TBCs with peroxidase activity (white arrowheads). Inset: nematocyte with moon-shape nucleus and an actin-dense V-shape structure (white) at the nematocyst apex (arrowhead). Yellow arrows point to fuzzy nematocysts. (E) Disorganized Zic4/Sp5(RNAi) tentacle, containing both typical TBCs (yellow outline) with SN (white arrows) and stenoteles (black arrows) and transforming TBCs (red dotted line) with few SNs and a granular cytoplasm (yellow arrowheads). (F) Co-detection of nematocilin- $A$ (purple) and Crim-1 (pink) in macerates as indicated. Yellow square: enlarged cell; yellow arrows: SNs; blue arrows: LNs. (G) Peroxidase activity in BDCs and TBCs from basal and apical regions from $H v \_A E P 2$ animals treated as indicated, dissected and trypsin macerated. 

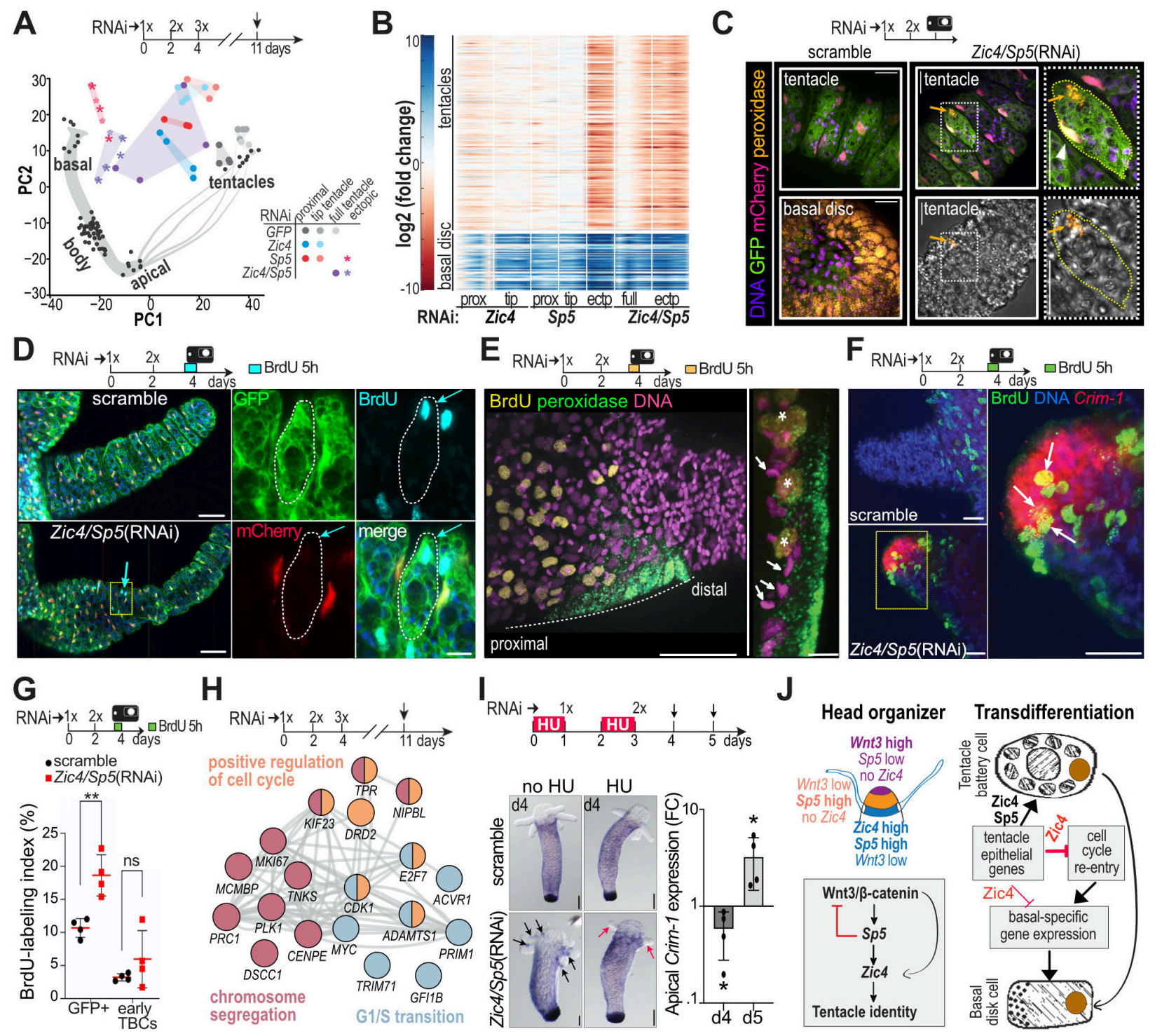

Figure 4. Transdifferentiating tentacle battery cells re-enter the cell cycle.

(A) RNA-seq performed on dissected tentacles as indicated and projection into a PCA-space, generated by positional RNA-seq. (B) Heatmap showing differential expression of tentacle/basal disc markers. Full: whole tentacles, Prox, tip: proximal/distal tentacle regions, ectp: ectopic structures. (C) Detection of peroxidase (arrows) in FUCCI-eGFP animals. (D) BrdU+ TBC (blue arrows) enlarged on the right (white outline). (E) Peroxidase+ droplets (green) in BrdU+ TBC; right: enlarged section along the dotted line, asterisks: BrdU+ peroxidase + cells (yellow), white arrows: nematocyte nuclei. (F) Crim-1+ BrdU+ tentacle cells (white arrows). (G) BrdU-labeling index in apical epithelial cells (GFP+) and early TBCs. (H) Subnetwork of cell cycle genes upregulated in tentacles across all RNAi conditions (depicted in panel B); color-coding indicates GO terms, edges genetic/physical interactions, co-localization and/or co-expression. (I) Left: Ectopic Crim-1 (black arrows) no longer detected after HU-treatment (red arrows). Right: Crim-1 qPCR. (J) Left: Head organizer defined by Wnt3/ Sp5/Zic4 domains and signaling cascade for tentacle identity. Right: Transdifferentiation model. P-values: $* * \leq 0.01, *<0.05$. Error bars 
bioRxiv preprint doi: https://doi.org/10.1101/2021.12.22.473838; this version posted December 23, 2021. The copyright holder for this preprint (which was not certified by peer review) is the author/funder, who has granted bioRxiv a license to display the preprint in perpetuity. It is made available under aCC-BY-NC-ND 4.0 International license.

indicate SD. Scale bars: $25 \mu \mathrm{m}(\mathrm{C}, \mathrm{F}), 50 \mu \mathrm{m}$ (D) $10 \mu \mathrm{m}$ for enlargement, $50 \mu \mathrm{m}$ (E) $20 \mu \mathrm{m}$ for enlargement, $200 \mu \mathrm{m}(\mathrm{I})$. 